\title{
Gestational diabetes induces alterations in the function of neonatal endothelial colony-forming cells
}

\author{
Emily K. Blue 1,2, Robert DiGiuseppe', Ethel Derr-Yellin ${ }^{1,2}$, Juan Carlos Acosta', S. Louise Pay ${ }^{2}$, Helmut Hanenberg ${ }^{1-3}$, \\ Megan M. Schellinger ${ }^{4}$, Sara K. Quinney ${ }^{4}$, Julie A. Mund ${ }^{1-3}$, Jamie Case ${ }^{1-3}$ and Laura S. Haneline ${ }^{1-3,5,6}$
}

BACKGROUND: Children born to mothers with gestational diabetes mellitus (GDM) experience increased risk of developing hypertension, type 2 diabetes mellitus, and obesity. Disrupted function of endothelial colony-forming cells (ECFCs) may contribute to this enhanced risk. The goal of this study was to determine whether cord blood ECFCs from GDM pregnancies exhibit altered functionality.

METHODS: ECFCs isolated from the cord blood of control and GDM pregnancies were assessed for proliferation, senescence, and Matrigel network formation. The requirement for p38MAPK in hyperglycemia-induced senescence was determined using inhibition and overexpression studies.

RESULTS: GDM-exposed ECFCs were more proliferative than control ECFCs. However, GDM-exposed ECFCs exhibited decreased network-forming ability in Matrigel. Aging of ECFCs by serial passaging led to increased senescence and reduced proliferation of GDM-exposed ECFCs. ECFCs from GDM pregnancies were resistant to hyperglycemia-induced senescence compared with those from controls. In response to hyperglycemia, control ECFCs activated P38MAPK, which was required for hyperglycemia-induced senescence. In contrast, GDMexposed ECFCs showed no change in p38MAPK activation under equivalent conditions.

CONCLUSION: Intrauterine exposure of ECFCs to GDM induces unique phenotypic alterations. The resistance of GDMexposed ECFCs to hyperglycemia-induced senescence and decreased p38MAPK activation suggest that these progenitor cells have undergone changes that induce tolerance to a hyperglycemic environment.

G estational diabetes mellitus (GDM) is common and complicates $\sim 5-10 \%$ of all pregnancies (1). Substantial rates of maternal and neonatal complications are associated with GDM. However, in addition to short-term neonatal morbidities, multiple studies demonstrate that offspring of mothers with GDM have an increased risk of developing several chronic diseases, including hypertension, obesity, type 2 diabetes, and the metabolic syndrome (2-5). Despite evidence linking prenatal exposure to GDM with several diseases later in life, the mechanism for disease pathogenesis is currently unknown.

Individuals with diabetes mellitus (DM) are at a high risk of developing micro- and macrovascular diseases, demonstrating that a diabetic environment promotes vascular injury (6). Endothelial dysfunction precedes the development of many vascular diseases (7). The repair of damaged endothelium requires intact function of progenitor cells that reside in vessel walls and that circulate in the peripheral blood $(8,9)$. Studies in adults and adolescents with DM show that reduced numbers and altered function of circulating progenitors are associated with endothelial dysfunction (10,11). Given these observations, we hypothesize that fetal exposure to a diabetic environment accelerates the onset of endothelial dysfunction by altering endothelial progenitor quantity and function.

Studies by our group and by others have characterized highly proliferative circulating endothelial progenitor cells, referred to as endothelial colony-forming cells (ECFCs) $(8,9,12,13)$. Previously, we demonstrated that ECFCs from the cord blood of infants born to women with pregestational DM have reduced colony formation, premature senescence, decreased proliferation, and decreased vessel-forming ability compared with control cord blood ECFCs (13). Because ECFCs from neonates born to women with GDM are also exposed to the diabetic milieu in utero, we hypothesized that cord blood ECFCs from GDM pregnancies would also exhibit altered function.

\section{RESULTS}

\section{ECFCs From GDM Pregnancies Exhibit Increased Proliferation} and Reduced Tube Formation

The ability of a single cell to form a visible colony in vitro is a key defining characteristic of progenitor cells. Therefore, ECFCs from the cord blood of control and GDM pregnancies were plated in limiting dilution assays to validate progenitor function via colony-forming ability. In contrast with our previous data on ECFCs from pregnancies complicated by pregestational DM (13), GDM-exposed and control ECFCs, from both original cord blood samples (Figure 1a) and established

\footnotetext{
'Department of Pediatrics, Indiana University School of Medicine, Indianapolis, Indiana; ${ }^{2}$ Herman B Wells Center for Pediatric Research, Indiana University School of Medicine, Indianapolis, Indiana; ${ }^{3}$ Indiana University Simon Cancer Center, Indiana University School of Medicine, Indianapolis, Indiana; ${ }^{4}$ Department of Obstetrics and Gynecology, Indiana University School of Medicine, Indianapolis, Indiana; ${ }^{5}$ Department of Microbiology and Immunology, Indiana University School of Medicine, Indianapolis, Indiana; ${ }^{6}$ Department of Cellular and Integrative Physiology, Indiana University School of Medicine, Indianapolis, Indiana. Correspondence: Laura S. Haneline (Ihanelin@iu.edu) 
a
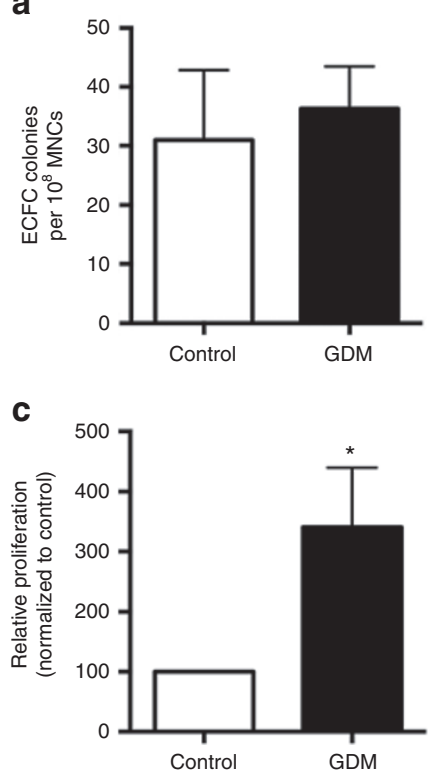

b

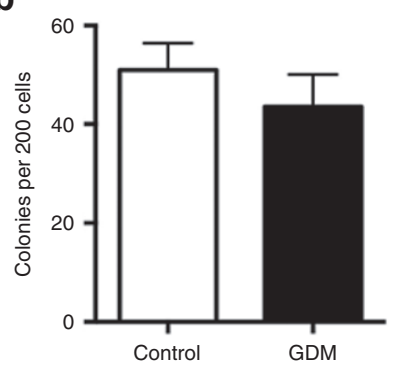

d

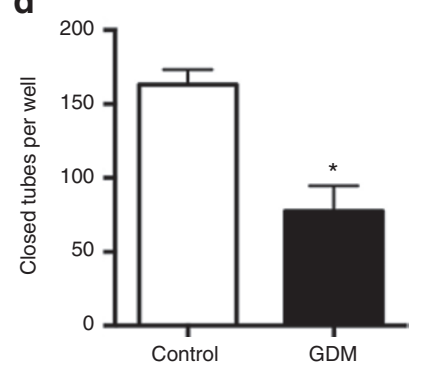

Figure 1. Gestational diabetes mellitus (GDM)-exposed endothelial colony-forming cells (ECFCs) exhibit increased proliferation and impaired tube-forming ability. (a) Frequency of ECFC colonies per $10^{8}$ cord blood mononuclear cells (MNCs). Approximately $2 \times 10^{8}$ MNCs were plated on collagen-coated plates. Colonies were counted between d 10 and 14. Frequency of ECFCs was calculated by normalizing to cell number plated for each sample; $n=5$ for control and $n=7$ for GDM samples. (b) Frequency of ECFC colonies in limiting dilution assays. Colonies were scored by visual inspection after $7 \mathrm{~d}$ in culture; $n=6$ per group. (c) ${ }^{3} \mathrm{H}$-Thymidine incorporation assay to assess proliferation. Relative proliferation was calculated by dividing the scintillation count for individual cultures by the baseline value for control ECFCs and then multiplying by 100 . Results are from seven independent experiments using five to six different cell lines per group. White bars indicate control ECFCs, and black bars indicate GDM-exposed ECFCs; ${ }^{*} P<0.05$ by Wilcoxon signed-rank test. (d) Network formation in Matrigel. ECFCs were plated in Matrigel for $16 \mathrm{~h}$. Images were obtained, and total closed networks per well were quantified; $n=4$ per group; ${ }^{*} P<0.05$ compared with control by unpaired $t$-test.

cell lines (Figure 1b), had similar colony-forming abilities. To determine whether intrauterine exposure to GDM altered ECFC function, in vitro assays were performed to assess the following: senescence by staining for senescence-associated $\beta$-galactosidase (14), proliferation using ${ }^{3} \mathrm{H}$-thymidine incorporation assays, and network-forming ability in Matrigel studies. Baseline levels of senescence were negligible in both GDM-exposed and control ECFCs (data not shown). However, proliferation of GDM-exposed ECFCs was significantly increased compared with that in controls (Figure 1c).

To further examine ECFCs from GDM pregnancies for evidence of dysfunction, cells were plated on Matrigel extracellular matrix to assess their ability to form closed network structures in vitro. Compared with control ECFCs, GDM-exposed ECFCs made $~ 50 \%$ fewer closed networks (Figure 1d). Taken together, these findings are intriguing, given that ECFCs from GDM pregnancies were hyperproliferative, yet had poor network formation in Matrigel studies. We questioned whether the enhanced proliferation observed in ECFCs from GDM pregnancies predisposes the cells to premature senescence.
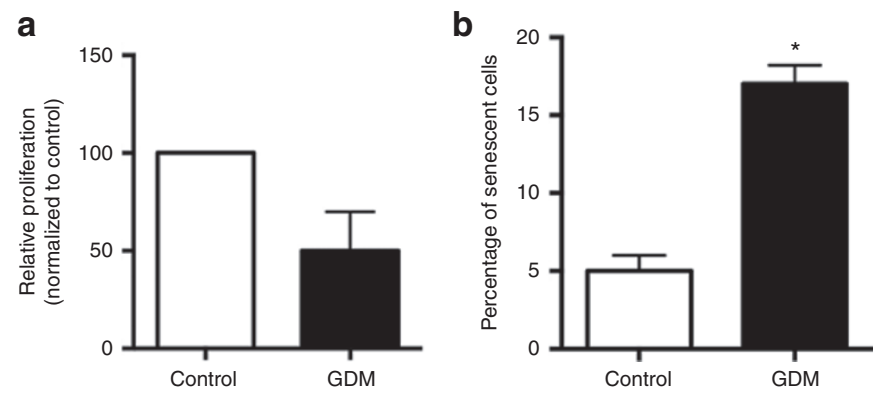

Figure 2. Serial passage of endothelial colony-forming cells (ECFCs) from gestational diabetes mellitus (GDM) pregnancies decreased proliferation and increased senescence. (a) ${ }^{3} \mathrm{H}$-Thymidine incorporation assay to assess proliferation, as described previously (13). Relative proliferation was calculated by dividing the scintillation count for individual cultures by the baseline value for control ECFCs and then multiplying by $100 ; n=4$. White bars indicate control ECFCs, and black bars indicate GDM-exposed ECFCs. Serial-passaged GDM-exposed ECFCs were no longer more proliferative than similarly passaged control ECFCs (not significant by unpaired $t$-test). (b) Senescence after serial passage. Frequency of senescence-associated $\beta$-galactosidase-positive cells was measured in passage 7 ECFCs; $n=3$; ${ }^{*} P<0.05$ by unpaired $t$-test.

Aging of cells to induce senescence can be modeled in vitro via serial replating. Therefore, we next asked whether higherpassage GDM-exposed ECFCs retained their hyperproliferative state and/or became senescent. Serial replating of ECFCs from GDM pregnancies showed that these cells had a reduced proliferative response. As shown in Figure 2a, higher-passage GDM-exposed ECFCs were no longer hyperproliferative and tended toward decreased proliferation. Senescence-associated $\beta$-galactosidase assays confirmed that serial replating of GDM-exposed ECFCs enhanced senescence compared with the result from the control ECFCs (Figure $2 b$ ). The increase in proliferation of early-passage GDM-exposed ECFCs may lead to subsequent premature senescence and reduced proliferation.

\section{ECFCs From GDM Pregnancies Are Resistant to}

\section{Hyperglycemia-Induced Senescence}

Previously, we demonstrated that control ECFCs exhibit enhanced senescence when exposed to hyperglycemia in vitro (13). It is possible that ECFCs from GDM pregnancies also undergo senescence in response to hyperglycemia. Alternatively, because ECFCs from GDM pregnancies were exposed to hyperglycemia in utero and have no increase in baseline senescence, GDM-exposed ECFCs may have adapted to the deleterious effects of hyperglycemia. To determine whether ECFCs from GDM pregnancies undergo senescence following treatment with elevated glucose concentrations, control and GDM-exposed ECFCs were cultured in euglyce$\mathrm{mic}(5 \mathrm{mmol} / \mathrm{l}$ glucose) and hyperglycemic (10 or $15 \mathrm{mmol} / \mathrm{l}$ glucose) conditions. As shown in Figure $3 \mathrm{a}$ and similar to the results of previous studies (13), treating control ECFCs with either 10 or $15 \mathrm{mmol} / \mathrm{l}$ glucose induced a greater frequency of senescent cells. However, exposing ECFCs from GDM pregnancies to hyperglycemia did not increase senescence, resulting in significantly lower senescence compared with that 
a

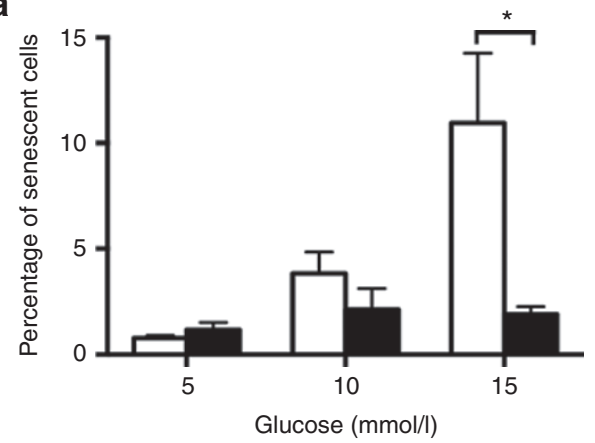

b

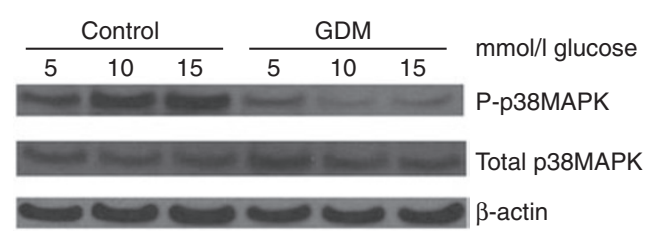

C

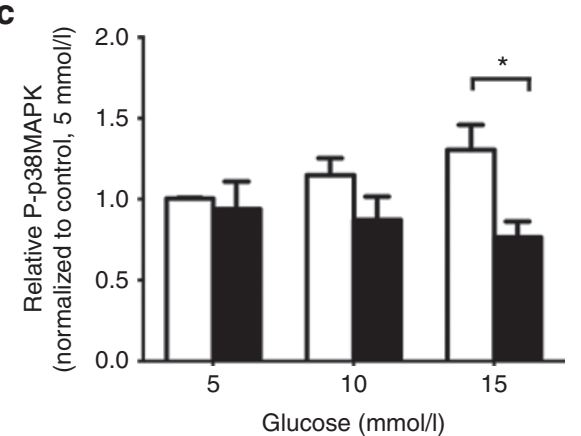

d

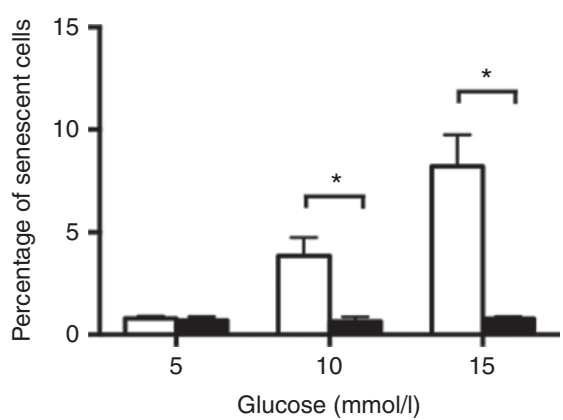

Figure 3. Hyperglycemic treatment of gestational diabetes mellitus (GDM)-exposed endothelial colony-forming cells (ECFCs) did not induce senescence or activate p38MAPK. (a) Hyperglycemia-induced senescence. ECFCs were plated in endothelial cell growth medium EGM2 containing the indicated glucose concentration for $7 \mathrm{~d}$. The percentage of senescence-associated $\beta$-galactosidase (SA- $\beta$-gal)-positive cells was calculated in four independent experiments. White bars indicate control ECFCs, and black bars indicate GDM-exposed ECFCs. Two-way ANOVA determined a significant increase in senescence by hyperglycemia, with significantly less senescence in GDM-exposed ECFCs. ${ }^{*} P<0.05$ by Sidak's multiple comparison. (b) Hyperglycemia also induced p38MAPK phosphorylation in ECFCs. Western blotting was performed on lysates from control ECFCs treated with indicated glucose concentrations to detect phospho-p38MAPK (P-p38MAPK) and total p38MAPK. $\beta$-actin was used as a loading control. (c) Quantitation of band intensity from western blots in panel (b) in six independent experiments. Bands were quantified in ImageJ. P-p38MAPK levels were normalized to those of total p38MAPK. A significant increase in p38MAPK phosphorylation with higher glucose concentration was found by two-way ANOVA. Decreased P-p38MAPK was found in GDM-exposed ECFCs, as indicated $\left({ }^{*} P<0.05\right)$ by Sidak's multiple comparisons. (d) Effect in control cells. In addition, culturing ECFCs in elevated glucose concentrations did not increase the numbers of apoptotic or necrotic cells among the control or GDM-exposed ECFCs (data not shown). These data indicate that GDM-exposed ECFCs are resistant to hyperglycemia-induced senescence and suggest that adaptive changes that protect from hyperglycemic stress have occurred.

Previous studies have demonstrated that $\mathrm{p} 38 \mathrm{MAPK}$ is required for hyperglycemia-induced senescence in adult endothelial progenitor cells $(15,16)$. However, the role of p38MAPK in promoting senescence of neonatal ECFCs after hyperglycemic exposure is unknown. Therefore, our initial studies evaluated whether control ECFCs treated with 10 or $15 \mathrm{mmol} / \mathrm{l}$ glucose increased p38MAPK activation. Hyperglycemic treatment of control ECFCs stimulated a dose-dependent increase in phosphorylated or active p38MAPK (Figure 3b,c). To determine whether ECFC senescence was dependent on p38MAPK activation, control ECFCs were cultured with or without a p38MAPK inhibitor, SB203580, in euglycemic and hyperglycemic conditions. Inhibition of p38MAPK prevented hyperglycemia-induced senescence in control ECFCs (Figure 3d), supporting an integral role for this stress-activated pathway in regulating senescence of ECFCs.

Given that p38MAPK is required for hyperglycemiainduced senescence, together with the observation that GDM-exposed ECFCs do not undergo senescence after hyperglycemic treatment, we speculated that activation of p38MAPK was impaired in ECFCs from GDM pregnancies. As shown in Figure $3 b$ and quantified in Figure $3 c$, highglucose treatment of ECFCs from GDM pregnancies did not increase p38MAPK phosphorylation. These results are consistent with the idea that $\mathrm{p} 38 \mathrm{MAPK}$ is required for hyperglycemia to induce senescence in ECFCs and indicate that resistance of GDM-exposed ECFCs to hyperglycemia-induced senescence may be due to aberrant regulation of p38MAPK activation.

To confirm a role for the p38MAPK pathway in regulating senescence of ECFCs, mutant CDNAs of mitogen-activated protein kinase kinase 6 (MKK6) were stably expressed in ECFCs using lentiviral transduction methods (17). MKK6 was chosen because it directly and specifically activates p38MAPK by phosphorylating residues in the activation loop (18). Initially, a control ECFC cell line was transduced with lentiviral vectors encoding an empty vector, a constitutively active MKK6, or an inactive MKK6 construct. Western blotting confirmed MKK6 overexpression in cells transduced with lentiviruses encoding the mutant MKK6 cDNAs and verified increased phosphorylation of p38MAPK in cells overexpressing constitutively active MKK6 (Figure 4a). For subsequent studies, control and GDM-exposed ECFC cell lines expressing either the constitutively active or inactive MKK6 constructs were evaluated for

of p38MAPK inhibition on senescence. Control ECFCs were cultured in 5, 10 , or $15 \mathrm{mmol} / \mathrm{l}$ glucose with or without $1 \mu \mathrm{mol} / \mathrm{l}$ of the $\mathrm{P} 38 \mathrm{MAPK}$ inhibitor SB203580. White bars indicate control ECFCs, and black bars indicate GDM-exposed ECFCs. After $7 d$, the percentage of SA- $\beta$-gal-positive cells was quantified. Results are from four independent experiments. SB203580 significantly decreased senescence, with $* P<0.05$, as indicated (two-way ANOVA, followed by Sidak's multiple comparisons). 
a

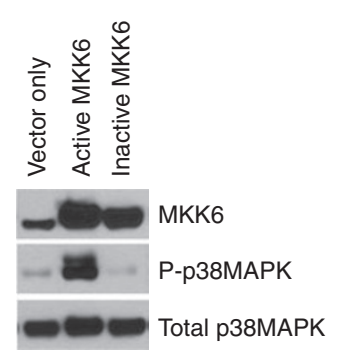

C

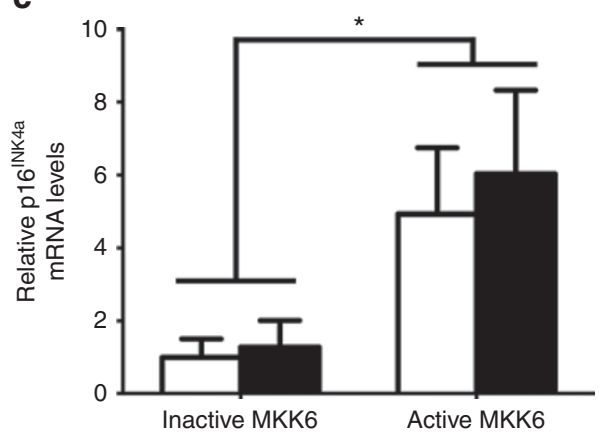

b

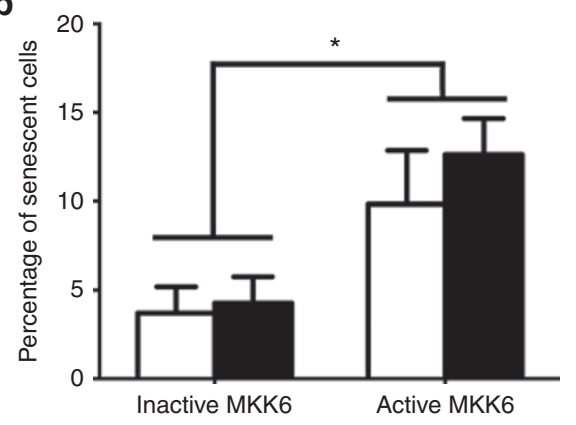

d

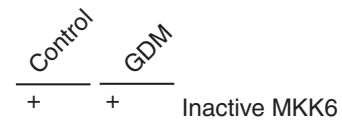

$+\quad+$ Active MKK6

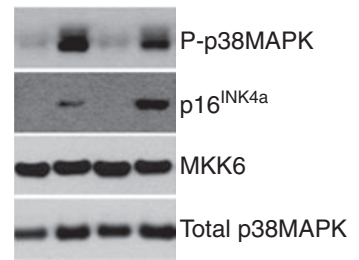

Figure 4. Activation of p38MAPK in exposed endothelial colony-forming cells (ECFCs) induces senescence and p16 expression. (a) Overexpression of active mitogen-activated protein kinase kinase 6 (MKK6) by lentiviral transduction in control ECFCs increases phospho-p38MAPK (P-p38MAPK). Control ECFCs were also transduced by lentivirus expressing empty vector or kinase-inactive MKK6. Western blotting of lysates confirmed increased phosphorylation of p38MAPK by active MKK6, but not by the empty vector or inactive MKK6. Blot is representative of three independent experiments. (b) Effect of p38MAPK activation by active MKK6 on senescence in control and GDM-exposed ECFCs. Following transduction with either active or inactive MKK6, the percentage of senescence-associated $\beta$-galactosidase (SA- $\beta$-gal)-positive control and gestational diabetes mellitus (GDM)-exposed ECFCs was assessed. White bars indicate control ECFCs, and black bars indicate GDM-exposed ECFCs. Expression of active MKK6 induced significantly increased senescence in both control and GDM-exposed ECFCs, as determined by two-way ANOVA ( ${ }^{*} P<0.05$ ). No differences between control and GDM-exposed ECFCs were found by Sidak's multiple comparisons. Results shown are from three independent experiments. (c) p16 $16^{\text {INAa }}$ expression is increased following expression of active MKK6, but not of inactive MKK6. RNA was isolated from control and GDM-exposed ECFCs overexpressing active or inactive MKK6. Relative mRNA levels of p16 ${ }^{\text {INK4a }}$ were quantified by real-time reverse-transcriptase $P C R$, normalized to hypoxanthine phosphoribosyltransferase expression. White bars indicate control ECFCs, and black bars indicate GDM-exposed ECFCs. Two-way ANOVA identified a significant increase in p16 $6^{\text {INK4a }}$ mRNA in ECFCs overexpressing active MKK6 compared with ECFCs expressing inactive MKK6 ( $\left.{ }^{*} P<0.05\right)$. No significant difference was found between control and GDM-exposed ECFCs. Results represent three different cell lines from both control and GDM groups. (d) Western blotting confirmed increased expression of p16 $6^{\text {INK } 4 a}$ with active MKK6 and p38MAPK activation (P-p38MAPK formation). Total p38MAPK is shown as a loading control. Blots are representative of three independent experiments.

senescence under standard culture conditions. Both control and GDM-exposed ECFCs expressing constitutively active MKK6 had increased senescent cells compared with ECFCs expressing inactive MKK6 (Figure $4 \mathbf{b}$ ). Previous studies demonstrated that the molecule downstream of p38MAPK required for promoting a senescent cellular fate is $\mathrm{p} 16^{\mathrm{INK} 4 \mathrm{~A}}$, a cyclin-dependent kinase inhibitor (19). Therefore, we next examined transduced cells for $\mathrm{p} 16^{\mathrm{INK} 4 \mathrm{~A}}$ expression by reversetranscriptase PCR and western blotting. In control and GDMexposed ECFCs with increased p38MAPK activity, p16 $6^{\text {INK4A }}$ expression was significantly elevated at the RNA and protein levels compared with that in controls expressing the inactive MKK6 mutant (Figure 4c,d). Collectively, these data support an important role for the p38MAPK pathway in regulating senescence of ECFCs, in addition to supporting the aberrant regulation of $\mathrm{p} 38 \mathrm{MAPK}$ in ECFCs from GDM pregnancies.

\section{DISCUSSION}

Infants exposed to either GDM or pregestational DM in utero are at increased risk of developing several chronic diseases, and in some cases, with onset of symptomatology during childhood $(2-5)$. Increases in systolic blood pressure and adiposity can be detected as early as $3 \mathrm{y}$ of age in children born to women with GDM (2). Furthermore, offspring of pregnancies complicated by either GDM or pregestational DM exhibit an enhanced risk for childhood onset of elevated blood pressure, overt hypertension, type 2 diabetes, and obesity $(3,5)$. These complications have immediate health consequences for individual children. Moreover, each of these morbidities constitutes a significant risk factor for eventual cardiovascular mortality. Thus, it is critical to identify underlying mechanisms involved in disease pathogenesis so that preventative measures can be instituted prospectively in children of mothers with DM.

Fetal exposure to an adverse intrauterine environment probably results in alterations in long-lived stem and progenitor cells, especially given the early acquisition of chronic diseases that are typically found in older adults by the offspring. The model of stem and/or progenitor cell damage and subsequent dysfunction as a mechanism involved in vascular disease progression has been described previously $(20,21)$. However, 


\section{Articles | Blueetal.}

minimal data are reported to support this concept in the developmental origins of vascular disease. ECFCs, an important progenitor population critical for vascular homeostasis, circulate in adult peripheral blood but are highly enriched in the endothelium of vessel walls and in umbilical cord blood (8). Circulating levels of ECFCs are altered in patients with coronary artery disease and diabetic retinopathy $(12,22,23)$. Healthy adolescents and young adults with type $1 \mathrm{DM}$ have altered circulating levels of ECFCs and evidence of endothelial dysfunction (11). Importantly, pulmonary artery ECFCs from patients with pulmonary artery hypertension exhibit increased proliferation (24). Based on these previous studies correlating ECFC levels and function with vascular disease, ECFCs represent a novel and accessible progenitor population to study the effects of an adverse intrauterine environment.

Previously, to test this notion, we examined ECFCs harvested from pregnancies complicated by pregestational DM for functional impairments. Pregestational DM-exposed ECFCs had decreased colony-forming ability, reduced proliferative capacity, increased basal senescence, and profound deficiencies in vasculogenesis (13). In this study, we examined whether ECFCs from GDM pregnancies would be less dysfunctional because GDM is often associated with milder diabetic symptoms for a shorter duration compared with the symptoms of pregestational DM (25). Although several progenitor phenotypes examined support this concept (i.e., normal colony formation, normal baseline senescence, and modest reduction in tube-forming ability), an unexpected finding was hyperproliferation of GDM-exposed ECFCs. We speculate that this observation may reflect an adaptive response of ECFCs following intrauterine exposure to a GDM environment. However, increased proliferation was only transient. Passaging of GDMexposed ECFCs led to decreased proliferative responses and premature senescence, which are remarkably similar to phenotypes observed in ECFCs from pregnancies complicated by type 1 and type $2 \mathrm{DM}$. It is possible that ECFCs exposed to pregestational DM may also exhibit a compensatory period of hyperproliferation. Due to the greater severity and/or longer exposure to the intrauterine DM environment, neonatal ECFCs harvested from pregestational DM pregnancies may have already progressed toward reduced proliferation and enhanced senescence. Future studies in animal models could be used to test this hypothesis, given the lack of feasibility in evaluating fetal ECFCs from human subjects.

Another adaptation identified in GDM-exposed ECFCs was resistance to hyperglycemia-induced senescence. The tolerance to hyperglycemia probably occurs through a signaling alteration involving p38MAPK, which is activated and required for senescence of control ECFCs. GDM-exposed ECFCs treated with hyperglycemia did not activate p38MAPK, allowing the cells to resist senescence and to continue proliferating in the presence of elevated glucose concentrations. GDM-exposed ECFCs were capable of phosphorylating p38MAPK and undergoing senescence when an upstream activating molecule, MKK6, was expressed. Together, these data support dysregulation of p38MAPK activity in ECFCs from GDM pregnancies, rather than an intrinsic defect in the ability of the ECFCs to undergo senescence. Although further studies are needed to elucidate the underlying mechanism responsible for aberrant p38MAPK regulation, our data identified novel cellular and biochemical adaptations in a primary neonatal progenitor population after in vivo GDM exposure. Our findings and approach have advantages compared with previous studies that examined biochemical and molecular alterations of endothelial cells treated with hyperglycemia in vitro $(16,26,27)$ because the neonatal impact of GDM exposure is challenging to model in vitro or to replicate in animal models. This is due to the complexity of GDM, which involves maternal insulin resistance, $\beta$-cell dysfunction, hyperlipidemia, and inflammation, resulting in fetal hyperglycemia, hyperinsulinemia, and presumably additional metabolic consequences yet to be identified (28). Therefore, studying ECFCs after in vivo GDM exposure enhances physiological relevance and provides unique insight into the long-term effects of the overall diabetic milieu on a neonatal progenitor cell population.

\section{METHODS}

\section{Cord Blood Sample Collection}

Umbilical cord blood samples were collected from healthy, control pregnancies and pregnancies complicated by GDM after informed consent was obtained from the mothers. All pregnancies were singleton gestations. GDM was defined according to the guidelines of the American College of Obstetrics and Gynecology (29). All control subjects had a normal 50 -g oral glucose screen. Women with preeclampsia or hypertension, women with other illnesses known to affect glucose metabolism, and women taking medications known to affect glucose metabolism were excluded. In addition, infants with known chromosomal abnormalities were excluded. The Institutional Review Board at the Indiana University School of Medicine approved this protocol. Pertinent health information is included for women (Table 1) and infants (Table 2) enrolled in the study.

\section{ECFC Culture and Isolation}

ECFCs were isolated from cord blood samples by the Indiana University Simon Cancer Center's Angiogenesis, Endothelial and Pro-Angiogenic Cell Core as previously described $(13,30)$. For routine culture of ECFCs, cells were grown in endothelial cell growth

Table 1. Clinical data for maternal subjects

\begin{tabular}{lclcc}
\hline & $\begin{array}{c}\text { Maternal } \\
\text { age (y) }\end{array}$ & Medications & $\begin{array}{c}\text { Maternal } \\
\text { prepregnancy BMI }\end{array}$ & HbA1c \\
\hline Control 1 & 26 & Penicillin & 48 & ND \\
Control 2 & 38 & None & 28 & ND \\
Control 3 & 26 & None & 44 & ND \\
Control 4 & 37 & None & 37 & ND \\
Control 5 & 42 & None & 35 & ND \\
Control 6 & 24 & Zoloft & 19 & ND \\
GDM 1 & 32 & None & 31 & 5.8 \\
GDM 2 & 28 & Insulin & 27 & 6.4 \\
GDM 3 & 24 & None & 44 & 6.2 \\
GDM 4 & 25 & None & 37 & ND \\
GDM 5 & 41 & Penicillin & 34 & 5.9 \\
GDM 6 & 33 & Glyburide, Zoloft & 39 & 5.6 \\
GDM 7 & 37 & None & 20 & 5.7 \\
\hline
\end{tabular}

GDM, gestational diabetes mellitus; $\mathrm{HbA} 1 \mathrm{c}$, glycosylated hemoglobin; ND, not done. 
Table 2. Clinical data for infant subjects

\begin{tabular}{|c|c|c|c|c|c|}
\hline & Sex & $\begin{array}{c}\text { Gestational age } \\
\text { (weeks completed) }\end{array}$ & Infant weight (kg) & $\begin{array}{l}\text { Infant weight } \\
\text { percentile }^{\mathrm{a}}(\%)\end{array}$ & $\begin{array}{l}\text { Infant weight/length } \\
\text { percentile }^{\mathrm{a}}(\%)\end{array}$ \\
\hline Control 1 & Male & 38 & 3.10 & $10-25$ & 25 \\
\hline Control 2 & Female & 40 & 3.38 & $25-50$ & $10-25$ \\
\hline Control 3 & Male & 41 & 3.98 & $75-90$ & $25-50$ \\
\hline Control 4 & Male & 39 & 3.70 & $50-75$ & $10-25$ \\
\hline Control 5 & Female & 38 & 2.91 & $10-25$ & $5-10$ \\
\hline Control 6 & Female & 40 & 3.45 & $50-75$ & $90-95$ \\
\hline GDM 1 & Male & 38 & 4.06 & $75-90$ & 50 \\
\hline GDM 2 & Female & 39 & 4.18 & $95-97$ & $95-97$ \\
\hline GDM 3 & Female & 39 & 3.76 & $75-90$ & $75-90$ \\
\hline GDM 4 & Male & 37 & 3.09 & $10-25$ & 5 \\
\hline GDM 5 & Female & 39 & 3.21 & $25-50$ & 25 \\
\hline GDM 6 & Male & 39 & 3.61 & $50-75$ & $50-75$ \\
\hline GDM 7 & Female & 38 & 3.47 & $50-75$ & $25-50$ \\
\hline
\end{tabular}

GDM, gestational diabetes mellitus.

aPercentiles obtained from Centers for Disease Control and Prevention (CDC) 2000 Clinical Growth Charts (http://www.cdc.gov/growthcharts/clinical_charts.htm).

medium EGM2 (Lonza, Walkersville, MD) containing 10\% fetal calf serum (FCS; Thermo Scientific Hyclone, Logan, UT)]. EGM2 is euglycemic $(5 \mathrm{mmol} / \mathrm{l})$. For hyperglycemia experiments, ECFCs were cultured in EGM2 supplemented with glucose to achieve concentrations of 10 or $15 \mathrm{mmol} / \mathrm{l}$. Early-passage ECFCs (less than passage 5) were used in all experiments, except where late-passage ECFCs are indicated (passage 7).

\section{Colony Formation Assays}

Each assay was performed in triplicate, plating 200 cells per well, as previously described (13). Average counts of total colonies from 5 to 7 cell lines from control and GDM groups are shown.

\section{${ }^{3} \mathrm{H}$-Thymidine Incorporation Assay}

Studies were performed as previously described (13). ECFCs were incubated in endothelial basal medium EBM2 containing 5\% FCS overnight and then plated in triplicate at 50,000 cells per well of a sixwell plate in EBM2 with 1\% FCS for $16 \mathrm{~h}$. ECFCs were then cultured in serum-free EBM2 for $8 \mathrm{~h}$ to induce quiescence. Proliferation was induced by addition of $2 \%$ FCS for $16 \mathrm{~h}$. Cells were pulsed with $1 \mu \mathrm{Ci}$ ${ }^{3} \mathrm{H}$-thymidine (Perkin-Elmer, Boston, MA) for $5 \mathrm{~h}$, washed, and then lysed in $0.1 \mathrm{~mol} / 1 \mathrm{NaOH}$. Incorporated ${ }^{3} \mathrm{H}$-thymidine was quantified on a liquid scintillation counter for triplicate wells. Average counts in four independent experiments were normalized to values obtained from control cells.

\section{Senescence-Associated $\beta$-Galactosidase Staining}

ECFCs were cultured in EGM2 containing the indicated concentration of glucose for $7 \mathrm{~d}$. Staining was performed as described $(13,14)$. Triplicate wells were plated for all conditions. At least 100 total cells per well were scored, and the average percentage of senescence-associated $\beta$-galactosidase-positive cells was determined per sample. Senescence was quantified for in vitro aged cells in three independent experiments and following hyperglycemia in four independent experiments.

\section{Matrigel Assay}

Tube-forming ability on Matrigel was evaluated as described (13). Briefly, 96-well plates were coated with $50 \mu \mathrm{l}$ Matrigel (Becton Dickenson, Franklin Lakes, NJ). ECFCs were plated in triplicate at a density of 5,000 cells per well in EGM2. After incubating at $37^{\circ} \mathrm{C}$ for $16 \mathrm{~h}$, phase contrast images were obtained using a Spot camera (Spot Imaging Solutions, Sterling Heights, MI) on an Axiovert 35 microscope (Zeiss, Thornwood, NY). The number of closed networks per well was scored. Triplicate measurements were averaged for each of four cell lines per group.

\section{Western Blotting}

Cells were lysed in radio-immunoprecipitation assay (RIPA) buffer containing Complete Protease Inhibitor Cocktail (Roche Applied Science, Indianapolis, IN). Equal amounts of protein were separated by sodium dodecyl sulfate polyacrylamide gel electrophoresis on precast gels (Life Technologies, Grand Island, NY). Protein was transferred to nitrocellulose, and immunoblotted with antibodies to phosphorylated p38MAPK, total p38MAPK, MKK6, (Cell Signaling Technology, Beverly, MA), p16 ${ }^{\mathrm{INK} 4 \mathrm{a}}$ (ab81278, Abcam, Cambridge, MA), or $\beta$-actin (Sigma-Aldrich, St. Louis, MO). Secondary antibodies conjugated to horseradish peroxidase were obtained from Biorad (Hercules, CA). Blots were developed with Pierce Supersignal West Pico (ThermoFisher, Hanover Park, IL), exposed to film, scanned, and compiled in Photoshop CS5.1 (Adobe, San Jose, CA). Band intensity was quantified using Image (NIH, Bethesda, MD). Graphs of semiquantitative data were generated on 3-6 cell lines per group.

\section{Generation of Lentivirus Encoding Recombinant MKK6 cDNA \\ Constructs}

Plasmids containing cDNA for a constitutively active MKK6 (Glu; Addgene plasmid 13518) or kinase-inactive MKK6 (K82A; Addgene plasmid 13519) were a generous gift from Roger Davis (18). The cDNAs were subcloned into the lentiviral vector pUC2CL6IPwo, which was obtained from Helmut Hanenberg (17). The lentiviral vector contains a puromycin resistance gene to enable selection of transduced cells. Lentivirus was produced by cotransfection of human embryonic kidney-293T cells with a lentiviral vector, packaging plasmid pCD/ NL-BH, and VSV-G envelope plasmid using Fugene 6 (Roche Applied Science). Lentivirus-containing supernatants were then collected, filtered through a $0.45-\mu \mathrm{mol} / \mathrm{l}$ asymmetric polyethersulfone filter unit (Thermo Scientific, Waltham, MA), and stored at $-80^{\circ} \mathrm{C}$.

\section{Lentiviral Transduction of ECFCs}

ECFCs were plated at 25,000 cells per well in a six-well tissue culture dish the day before transduction. Lentiviral vector supernatant was added to each well at dilutions of 1:5 to 1:10, in a final volume of $1 \mathrm{ml}$ of EGM2 + 10\% FCS containing $21 \mu \mathrm{mol} / \mathrm{l}(8 \mu \mathrm{g} / \mathrm{ml})$ Polybrene (SigmaAldrich), and incubated overnight. The medium was changed, and plates were then incubated for $48 \mathrm{~h}$. Transduced cells were selected in media containing $1.8 \mu \mathrm{mol} / \mathrm{l}(1 \mu \mathrm{g} / \mathrm{ml}$ ) puromycin (Life Technologies) for $2 \mathrm{~d}$ or until no live cells remained in the selection control plate. Protein lysates were obtained as described, and MKK6 expression and phosphorylated p38MAPK levels were confirmed for each transduction by western blotting. 


\section{Real-Time Reverse-Transcriptase PCR}

ECFCs were lysed in Qiazol (Qiagen, Valencia, CA), and RNA was obtained using the manufacturer's instructions. RNA was reverse transcribed using a Transcriptor Universal cDNA Master Kit (Roche Applied Science). Reverse-transcriptase PCR was performed on a Lightcycler 480 (Roche). For detection of p16 ${ }^{\text {INK4a }}$, a Universal Probe Master mix was used with Probe \#34 from the Roche Universal Probe Library, and p16 ${ }^{\mathrm{INK} 4 \mathrm{a}}$ primers (5'-GTGGACCTGGCTGAGGAG-3' and 5'-CTTTCAATCGGGGATGTCTG-3'). Hypoxanthine phosphoribosyltransferase was used to normalize $\mathrm{p} 16^{\text {INK4a }}$ values using the $2^{-\Delta \Delta \mathrm{Ct}}$ method. Detection of hypoxanthine phosphoribosyltransferase was performed using Lightcycler 480 SYBR Green I Master Mix (Roche) and the following hypoxanthine phosphoribosyltransferase primers: 5'-CCTTGGTCAGGCAGTATAATCCA-3'; 5'-GGTCCTTTTCACCAGCAAGCT-3').

\section{Statistical Analysis}

Data illustrated are mean \pm SEM. Two-way ANOVAs, followed by Sidak's multiple comparisons or Kruskal-Wallis one-way ANOVAs, were performed on comparisons of more than two groups, as indicated in the figure legends. Unpaired $t$-tests were conducted when only two groups were compared and data were normally distributed. Prism 6 (GraphPad Software, La Jolla, CA) was used for all statistical analyses, and significance was noted when $P<0.05$.

\section{ACKNOWLEDGMENTS}

The authors gratefully acknowledge research nurse Lucy Miller for assisting with cord blood collection. We thank Emily Sims and the Indiana University Simon Cancer Center's Angiogenesis, Endothelial and Pro-Angiogenic Cell Core for the processing and culture of the ECFCs from umbilical cord blood. We also thank Elizabeth Rybak for providing excellent administrative support.

\section{STATEMENT OF FINANCIAL SUPPORT}

US Public Health Services grants R01 HL094725 (L.S.H.), P30 CA82709 (L.S.H.), U10HD063094 (L.S.H. and S.Q.), P30DK090948 CEMH (J.C.), CA13823701 (H.H.), and CA155294-01 (H.H.); and the Riley Children's Foundation, Indianapolis, IN (L.S.H.).

Disclosure: The authors declare no conflicts of interest.

\section{REFERENCES}

1. Metzger BE, Buchanan TA, Coustan DR, et al. Summary and recommendations of the Fifth International Workshop-Conference on Gestational Diabetes Mellitus. Diabetes Care 2007;30:Suppl 2:S251-60.

2. Wright CS, Rifas-Shiman SL, Rich-Edwards JW, Taveras EM, Gillman MW, Oken E. Intrauterine exposure to gestational diabetes, child adiposity, and blood pressure. Am J Hypertens 2009;22:215-20.

3. Boney CM, Verma A, Tucker R, Vohr BR. Metabolic syndrome in childhood: association with birth weight, maternal obesity, and gestational diabetes mellitus. Pediatrics 2005;115:e290-6.

4. Bunt JC, Tataranni PA, Salbe AD. Intrauterine exposure to diabetes is a determinant of hemoglobin $\mathrm{A}(1) \mathrm{c}$ and systolic blood pressure in pima Indian children. J Clin Endocrinol Metab 2005;90:3225-9.

5. Cho NH, Silverman BL, Rizzo TA, Metzger BE. Correlations between the intrauterine metabolic environment and blood pressure in adolescent offspring of diabetic mothers. J Pediatr 2000;136:587-92.

6. Winer N, Sowers JR. Epidemiology of diabetes. J Clin Pharmacol 2004;44:397-405.

7. Avogaro A, Albiero M, Menegazzo L, de Kreutzenberg S, Fadini GP. Endothelial dysfunction in diabetes: the role of reparatory mechanisms. Diabetes Care 2011;34:Suppl 2:S285-90.

8. Ingram DA, Mead LE, Moore DB, Woodard W, Fenoglio A, Yoder MC. Vessel wall-derived endothelial cells rapidly proliferate because they contain a complete hierarchy of endothelial progenitor cells. Blood 2005;105:2783-6.

9. Ingram DA, Mead LE, Tanaka $\mathrm{H}$, et al. Identification of a novel hierarchy of endothelial progenitor cells using human peripheral and umbilical cord blood. Blood 2004;104:2752-60.

10. Georgescu A. Vascular dysfunction in diabetes: The endothelial progenitor cells as new therapeutic strategy. World J Diabetes 2011;2:92-7.
11. DiMeglio LA, Tosh A, Saha C, et al. Endothelial abnormalities in adolescents with type 1 diabetes: a biomarker for vascular sequelae? J Pediatr 2010;157:540-6.

12. Güven H, Shepherd RM, Bach RG, Capoccia BJ, Link DC. The number of endothelial progenitor cell colonies in the blood is increased in patients with angiographically significant coronary artery disease. J Am Coll Cardiol 2006;48:1579-87.

13. Ingram DA, Lien IZ, Mead LE, et al. In vitro hyperglycemia or a diabetic intrauterine environment reduces neonatal endothelial colony-forming cell numbers and function. Diabetes 2008;57:724-31.

14. van der Loo B, Fenton MJ, Erusalimsky JD. Cytochemical detection of a senescence-associated beta-galactosidase in endothelial and smooth muscle cells from human and rabbit blood vessels. Exp Cell Res 1998;241:309-15.

15. Kuki S, Imanishi T, Kobayashi K, Matsuo Y, Obana M, Akasaka T. Hyperglycemia accelerated endothelial progenitor cell senescence via the activation of p38 mitogen-activated protein kinase. Circ J 2006;70:1076-81.

16. Chen YH, Lin SJ, Lin FY, et al. High glucose impairs early and late endothelial progenitor cells by modifying nitric oxide-related but not oxidative stress-mediated mechanisms. Diabetes 2007;56:1559-68.

17. Nakano M, Kelly EJ, Wiek C, Hanenberg H, Rettie AE. CYP4V2 in Bietti's crystalline dystrophy: ocular localization, metabolism of $\omega-3-$ polyunsaturated fatty acids, and functional deficit of the p.H331P variant. Mol Pharmacol 2012;82:679-86.

18. Raingeaud J, Whitmarsh AJ, Barrett T, Dérijard B, Davis RJ. MKK3and MKK6-regulated gene expression is mediated by the p38 mitogenactivated protein kinase signal transduction pathway. Mol Cell Biol 1996;16:1247-55.

19. Lowe SW, Sherr CJ. Tumor suppression by Ink4a-Arf: progress and puzzles. Curr Opin Genet Dev 2003;13:77-83.

20. Olivieri F, Recchioni R, Marcheselli F, et al. Cellular senescence in cardiovascular diseases: potential age-related mechanisms and implications for treatment. Curr Pharm Des 2013;19:1710-9.

21. Minamino T, Komuro I. Vascular aging: insights from studies on cellular senescence, stem cell aging, and progeroid syndromes. Nat Clin Pract Cardiovasc Med 2008;5:637-48.

22. Tan K, Lessieur E, Cutler A, et al. Impaired function of circulating CD34(+) CD45(-) cells in patients with proliferative diabetic retinopathy. Exp Eye Res 2010;91:229-37.

23. Meneveau N, Deschaseaux F, Séronde MF, et al. Presence of endothelial colony-forming cells is associated with reduced microvascular obstruction limiting infarct size and left ventricular remodelling in patients with acute myocardial infarction. Basic Res Cardiol 2011;106:1397-410.

24. Duong HT, Comhair SA, Aldred MA, et al. Pulmonary artery endothelium resident endothelial colony-forming cells in pulmonary arterial hypertension. Pulm Circ 2011;1:475-86.

25. American Diabetes Association. Gestational diabetes mellitus. Diabetes Care 2004;27:Suppl 1:S88-90.

26. El-Osta A, Brasacchio D, Yao D, et al. Transient high glucose causes persistent epigenetic changes and altered gene expression during subsequent normoglycemia. J Exp Med 2008;205:2409-17.

27. Brasacchio D, Okabe J, Tikellis C, et al. Hyperglycemia induces a dynamic cooperativity of histone methylase and demethylase enzymes associated with gene-activating epigenetic marks that coexist on the lysine tail. Diabetes 2009;58:1229-36.

28. Hollingsworth DR. Alterations of maternal metabolism in normal and diabetic pregnancies: differences in insulin-dependent, non-insulin-dependent, and gestational diabetes. Am J Obstet Gynecol 1983;146:417-29.

29. ACOG Practice Bulletin. Clinical management guidelines for obstetriciangynecologists. Number 30, September 2001 (replaces Technical Bulletin Number 200, December 1994). Gestational diabetes. Obstet Gynecol 2001;98:525-38.

30. Mead LE, Prater D, Yoder MC, Ingram DA. Isolation and characterization of endothelial progenitor cells from human blood. Curr Protoc Stem Cell Biol 2008; Chapter 2:Unit 2C.1. 